\title{
Different Adsorption Behavior of Rare Earth and Metallic Ion Complexes on Langmuir Monolayers Probed by Sum-Frequency Generation Spectroscopy
}

\author{
Woongmo Sung ${ }^{1}$, David Vaknin ${ }^{2}$, and Doseok Kim ${ }^{1} *$ \\ ${ }^{1}$ Department of Physics, Sogang University, Seoul 121-742, Korea \\ ${ }^{2}$ Ames Laboratory and Department of Physics, Iowa State University, Ames, Iowa 50011, USA
}

(Received October 30, 2012 : revised January 14, 2013 : accepted January 16, 2013)

\begin{abstract}
Adsorption behavior of counterions under a Langmuir monolayer was investigated by sum-frequency generation (SFG) spectroscopy. By comparing SFG spectra of arachidic acid (AA) Langmuir monolayer/water interface with and without added salt, it was found that the simple trivalent cation $\mathrm{La}^{3+}$ adsorbed on $\mathrm{AA}$ monolayer only when the carboxylic headgroups are charged (deprotonated), implying that counterion adsorption is induced by Coulomb interaction. On the other hand, metal hydroxide complex $\mathrm{Fe}(\mathrm{OH})_{3}$ adsorbed even on a charge-neutral AA monolayer, indicating that the adsorption of iron hydroxide is due to chemical interaction such as covalent or hydrogen bonding to the headgroup of the molecules at the monolayer.
\end{abstract}

Keywords : Langmuir monolayer, Interfacial structure, Nonlinear optical spectroscopy

OCIS codes : (190.4350) Nonlinear optics at surfaces; (240.1485) Buried interfaces; (240.0310) Thin films

\section{INTRODUCTION}

Ions in cellular fluid are essential to virtually all processes of living organisms. For example, in the cell membrane of a neuron, the $\mathrm{K}^{+} / \mathrm{Na}^{+}$pump controls electric potential difference between the inner and the outer cell membrane in order to regulate important cellular functions such as muscle contraction, and neuron function [1]. $\mathrm{Mg}^{2+}$ makes a complex with ATP which carries chemical energy in the cell [2]. Unlike these well-known simple mono- and divalent cations, metal hydroxides such as $\mathrm{Fe}(\mathrm{OH})_{\mathrm{x}}{ }^{+3-x}$ are rarely found in living organisms. In 1975, Blakemore first found bacteria producing nanosized magnet filament [3]. These bacteria are called magneto-tactic bacteria (MTB) since they detect the Earth's magnetic field and tend to move toward it. As a natural plant for the production of magnetic nanoparticles, magneto-tactic bacteria have been recently studied by biologists and chemists. Tanaka et al. observed differences in size and crystal structure of nanoparticles by modifying a membrane protein which is supposed to be related to reduction of iron hydroxide [4]. Several previous studies suggested that the hydrophilic part of this membrane protein initiates nucleation of magnetic nanoparticles $[5,6]$. However, there was a still remaining ambiguity about which chemical group plays the major role in the nucleation process. Understanding the adsorption behavior of iron hydroxide at the molecular level can help to design or to modify the membrane proteins to control the size and crystal structure of magnetic nanoparticles.

To study the properties and functions of the cell membrane consisting of lipids and proteins, a monolayer formed at the air/water interface was commonly used as a model system [7, 8]. Due to their amphiphilic property, lipid molecules spontaneously make a mono-molecular thickness layer on a water surface where hydrophobic hydrocarbon chains and hydrophilic chemical groups are directed to the air and the water side, respectively. These monolayers are called Langmuir monolayers. In order to investigate the properties and structures of Langmuir monolayers, many optical techniques have been developed. For example, Brewster-angle microscopy (BAM) images a monolayer by measuring the refractive index difference between water and organic molecules at the surface [9]. Infrared reflection absorption spectroscopy (IRRAS) is able to detect vibrational modes of molecules at the surface monolayer [10]. But BAM gives only information of overall refractive index

\footnotetext{
*Corresponding author: doseok@sogang.ac.kr

Color versions of one or more of the figures in this paper are available online.
} 
change at the surface thus is not suitable to monitor the structure of a Langmuir monolayer and the interfacial water separately. And, IRRAS is not sensitive to interfacial water orientation. Therefore, a technique that can observe the subtle change of interfacial water structure expected from ion adsorption to the headgroups of the molecules in the Langmuir monolayer is required.

Sum-frequency generation (SFG) spectroscopy is a probe that ideally satisfies such requirements. As a second-order optical process, SFG is only allowed at the surface or interface where inversion symmetry is broken [11], and is sensitive to molecular orientation and conformation change. Since the invention of infrared-visible SFG spectroscopy by Y. R. Shen and coworkers, it has been widely used to investigate many different surfaces and interfaces. Structural differences between bulk and interfacial water were observed [12]. Surfaces of neat liquids and liquid mixtures were investigated $[13,14]$. Conformation change of biological model membranes with varying temperature, salt concentration, and $\mathrm{pH}$ condition was reported [15-18].

In this report, we used infrared-visible sum-frequency generation (SFG) spectroscopy to investigate the conformation of a biological model membrane consisting of arachidic acid $\left(\mathrm{CH}_{3}\left(\mathrm{CH}_{2}\right)_{18} \mathrm{COOH}\right)$, as well as water molecules beneath in the presence of multivalent ions in the subphase. Ion adsorption on this model Langmuir monolayer can be effectively monitored from the change in the sum-frequency spectra of interfacial water molecules.

\section{METHODS}

Figure 1 is a schematic description of our experimental setup. In the system, a picosecond Nd:YAG laser (PY-61C, Continuum Inc.) having $1064 \mathrm{~nm}$ wavelength, $\sim 15 \mathrm{~mJ} /$ pulse energy, and 50 ps pulsewidth was used as a light source. By using KTP crystal (CASIX), $532 \mathrm{~nm}$ visible light $(\sim 1 \mathrm{~mJ} /$

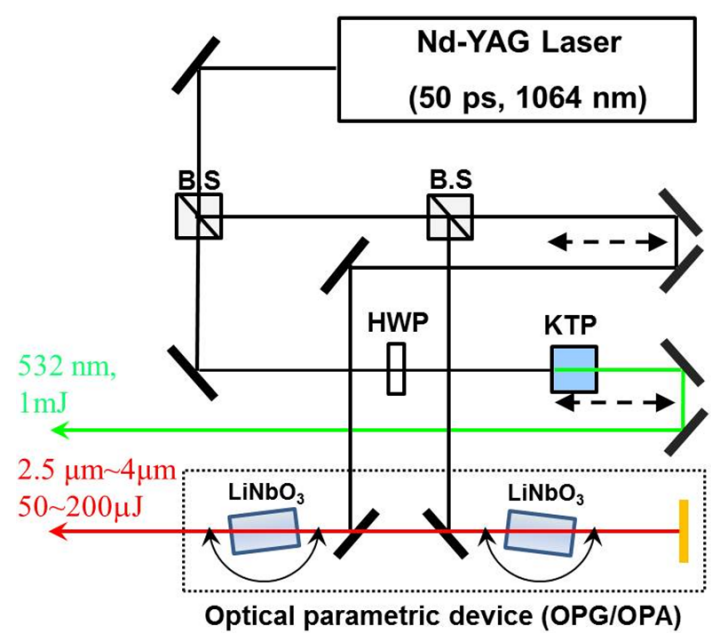

FIG. 1. Schematic diagram of SFG spectroscopy system. pulse) was generated by a type-II second harmonic generation process. The optical parametric generator/amplifier (OPG/OPA) part of the setup consists of two $\mathrm{LiNbO}_{3}$ crystals (CASIX) connected to the motorized stage. Rotation of the crystals changes the internal angle between the optic axis and the pump beam, and the mid-infrared signal $\left(5400 \mathrm{~cm}^{-1} \sim 6600 \mathrm{~cm}^{-1}\right)$ and idler $\left(2800 \mathrm{~cm}^{-1} \sim 4000 \mathrm{~cm}^{-1}\right)$ beams satisfying the type-I phase matching condition were generated. 2-mm thickness germanium plate was placed in the exit of the OPG/OPA in order to block the signal and the pump pulses. The energy of the IR idler at the sample stage was $\sim 150 \mu \mathrm{J} /$ pulse on average. Visible and the IR beams were incident on the samples at $45^{\circ}$ and $60^{\circ}$, respectively, and generated sum-frequency (SF) light in the reflection direction had an angle between $46.7^{\circ}$ and $47.3^{\circ}$. SF light was detected by photomultiplier tube (R928, Hamamatsu) and gated integrator (SR250, Stanford Research Systems). During the measurement, mean surface area of arachidic acid was maintained at $\sim 20 \mathrm{~A}^{2} /$ molecule, corresponding to liquid-condensed (LC) phase of the monolayer, by a homemade Teflon trough $(20 \mathrm{~cm} \times 10 \mathrm{~cm} \times 0.5 \mathrm{~cm})$ with a feedback loop from the surface pressure sensor (PS4, KSV NIMA) [8]. All experimental data was taken in SSP (S-polarization in SF signal, S-polarization in visible, and P-polarization in IR, respectively) polarization combination and normalized by SSP SF spectra from z-cut quartz crystal in order to compensate overlapping efficiency of visible and IR beams.

Both arachidic acid (purchased from Sigma) and hexadecanol (purchased from Aldrich) were prepared in chloroform solution $(\sim 3 \mathrm{mM}) .10$ minutes after spreading the stock solution on the water surface in the trough, the monolayer was compressed with a speed of $1 \mathrm{~A}^{2} / \mathrm{min}$. To avoid contamination by other organic molecules, the $\mathrm{pH}$ of the subphase solution was controlled only by $\mathrm{HCl}$ and $\mathrm{NaOH}$ without buffer. Afterward, $\mathrm{LaCl}_{3}$ and $\mathrm{FeCl}_{3}$ salts were introduced into the solution.

From the theory of SFG, the intensity of SF light can be represented as [13],

$$
\begin{aligned}
& I=\frac{8 \pi^{3} \omega_{S F}{ }^{2} \sec ^{2} \beta}{c^{3} n_{1}\left(\omega_{S F}\right) n_{1}\left(\omega_{V i s}\right) n_{1}\left(\omega_{I R}\right)}\left|\chi_{e f f}^{(2)}\right|^{2} I_{0}\left(\omega_{V i s}\right) I_{0}\left(\omega_{I R}\right) A T, \\
& \chi_{e f f}^{(2)}=\left[\hat{e}\left(\omega_{S F}\right) \cdot \stackrel{\leftrightarrow}{L}\left(\omega_{S F}\right)\right] \cdot\left[\overleftrightarrow{\chi^{(2)}}:\left[\stackrel{\leftrightarrow}{L}\left(\omega_{V i s}\right) \cdot \hat{e}\left(\omega_{V i s}\right)\right]\left[\stackrel{\leftrightarrow}{L}\left(\omega_{I R}\right) \cdot \hat{e}\left(\omega_{I R}\right)\right]\right],
\end{aligned}
$$

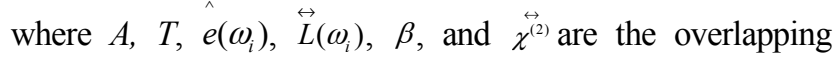
area of two input beams, input beam pulse width, unit vector in the polarization direction, tensor of the Fresnel factor, SF light angle in the reflection direction, and secondorder susceptibility tensor, respectively. In this equation, $\chi^{(2)}$ is determined by the characteristics of the surface, and is expressed using the second-order hyperpolarizability of the molecule as follows. 


$$
\chi^{(2)}{ }_{i j k}=N_{s} \sum_{\xi, \eta, \zeta} \alpha_{\xi \eta \zeta}^{(2)}<(\hat{\xi} \cdot \hat{i})(\hat{\eta} \cdot \hat{j})(\hat{\zeta} \cdot \hat{k})>
$$

where $N_{s}$ is the number density of molecules at the surface, and $\langle(\hat{\xi} \cdot \hat{i})(\hat{\eta} \cdot \hat{j})(\hat{\zeta} \cdot \hat{k})>$ indicates a direction cosine average between the laboratory coordinates $(i, j, k)$ and the molecular coordinates $(\xi, \eta, \zeta)$ [19]. Therefore, in the fixed beam geometry, SF intensity depends on both surface number density and relative orientation of molecules with respect to the experimental setup. For example, in the presence of static charges on top of the water surface, the water dipole can be reoriented along the E-field direction [20]. Since second order hyperpolarizability of the molecule has many vibrational resonant modes, it is represented as a sum over Lorentizian functions. Therefore, the intensity of SF spectra can be rewritten as [21],

$$
I=\left|A_{N R}+\sum_{q} \frac{A_{q}}{\omega_{I R}-\omega_{q}+i \Gamma_{q}}\right|^{2}
$$

where $A_{N R}$ is the non-resonant contribution, $A_{q}, \omega_{q}$, and $\Gamma_{q}$ are oscillation strength, resonant frequency, and damping constant of the $q$-th vibrational mode, respectively. This is the usual equation used to fit the experimental SF spectra, with which we will analyze our result for discussion in the next section.

\section{RESULT AND DISCUSSION}

First, we measured SF spectra of the arachidic acid monolayer/ water interface in the absence of salts as reference data. As shown in Fig. 2, there are two sharp peaks at $\sim 2875 \mathrm{~cm}^{-1}$ and $\sim 2935 \mathrm{~cm}^{-1}$ corresponding to symmetric

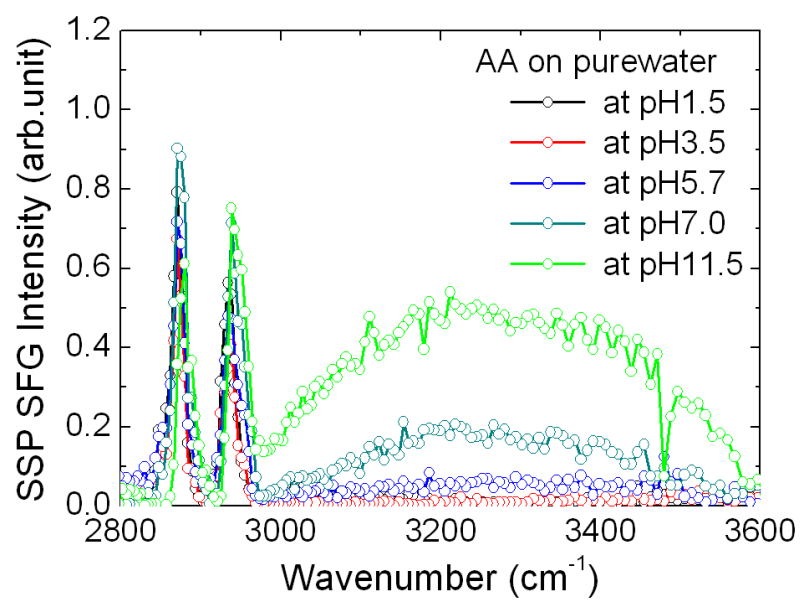

FIG. 2. SF spectra of arachidic acid monolayer on pure water at $\mathrm{pH} 1.5$ (black), 3.5 (red), 5.7 (blue), 7.0 (dark cyan), and 11.5 (green), respectively. All of the spectra were taken in SSP (S-polarization in SF signal, S-polarization in visible, and P-polarization in IR) polarization combination. stretch and Fermi resonance modes of $\mathrm{CH}_{3}$ terminal methyl groups of arachidic acid molecules, respectively [22]. In the spectra, the contribution from $\mathrm{CH}_{2}$ moieties in the alkyl chain, which is supposed to appear at $2850 \mathrm{~cm}^{-1}$ and $2920 \mathrm{~cm}^{-1}$, is not prominent, indicating the chains in the monolayer are in all-trans conformation. From $3000 \mathrm{~cm}^{-1}$ to $3600 \mathrm{~cm}^{-1}$, a broad SF band due to $\mathrm{OH}$-stretch modes of interfacial water was observed. Due to its strong hydrogen bonding nature, $\mathrm{OH}$-stretch mode of interfacial water is inhomogeneously broadened [23]. As $\mathrm{pH}$ of the solution increased above $\mathrm{pH} 5.7, \mathrm{SF}$ intensity of this $\mathrm{OH}$-stretch mode was considerably enhanced while spectral change in the $\mathrm{CH}_{\mathrm{x}}$ region is unnoticeable. This significant change in the $\mathrm{OH}$-stretch region originated from the alignment of water molecules induced by the static electric field from the headgroups. In more basic solution, the carboxyl headgroup of the arachidic acid monolayer tends to be deprotonated, carrying up to one negative unit charge per headgroup. As an alignment of water dipoles is made in the direction of the surface normal, it leads to the increase of direction cosine average in the second-order susceptibility in Eq. (2). Apparently, the spectral shape in the $\mathrm{CH}_{\mathrm{x}}$ region was also changed slightly in high $\mathrm{pH}$; for example, higher peak strength of $\mathrm{CH}_{3}$ fermi resonance than symmetric stretch mode. However this increase is due to an interference effect between the tail of the broad $\mathrm{OH}$ band and the $\mathrm{CH}_{\mathrm{x}}$ region. This apparent difference was also found in SF spectra of positively- and negatively-charged lipid monolayers on water, even when the resonance frequency, the peak strength, and the damping factor were similar for the two cases [21]. Therefore, the structure of the arachidic acid monolayer didn't seem to be disturbed much, even by repulsion between charged headgroups at high $\mathrm{pH}$.

Figure 3 shows SF spectra of an arachidic acid monolayer in the presence of $1 \mathrm{mM} \mathrm{LaCl}_{3}$ in the subphase water. Compared with Fig. 2, SF intensity came from $\mathrm{OH}$ vibrational mode of interfacial water was considerably smaller, and similar to charge-neutral monolayer (in the lower $\mathrm{pH}$

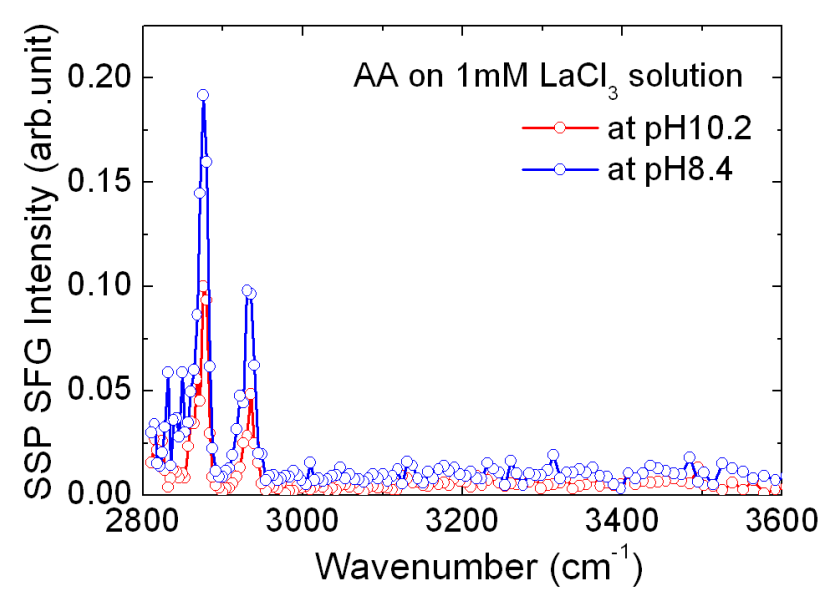

FIG. 3. SF spectra of arachidic acid monolayer on $1 \mathrm{mM}$ $\mathrm{LaCl}_{3}$ solution at $\mathrm{pH} 8.4$ (blue) and 10.2 (red). 
region in which the carboxylic headgroups of the AA molecules are not charged). It can be interpreted that on the negatively charged arachidic acid monolayer at high $\mathrm{pH}$, there are adsorbed $\mathrm{La}^{3+}$ counterions screening the surface electric field from the monolayer. We propose that on average one $\mathrm{La}^{3+}$ counterion is adsorbed to three negatively charged carboxyl groups to make a charge-neutral surface. From this result, it was found that adsorption of $\mathrm{La}^{3+}$ is dominantly induced by the coulomb interaction.

Next, we introduced $1 \mathrm{mM} \mathrm{FeCl}$ in pure water, and prepared an arachidic acid monolayer with the same mean surface area. In this experiment, two different ion compositions were considered. One is $1 \mathrm{mM}$ of $\mathrm{FeCl}_{3}$ dissolved in pure water initially in neutral $\mathrm{pH}(\sim 5.7)$. In this condition, most of the $\mathrm{Fe}^{3+}$ ions react with water molecules to form $\mathrm{Fe}(\mathrm{OH})_{3}$ to produce $\sim 3 \mathrm{mM}$ of $\mathrm{H}_{3} \mathrm{O}^{+}$. Measured $\mathrm{pH}$ of the solution was 2.6, confirming that more than $90 \%$ of $\mathrm{Fe}^{3+}$ ions turn into $\mathrm{Fe}(\mathrm{OH})_{3}$ and less than $10 \%$ of metal hydroxide $\mathrm{Fe}(\mathrm{OH})_{2}{ }^{+}$[24]. The other sample is the same amount of $\mathrm{FeCl}_{3}$ dissolved in a highly acidic solution. By adding diluted $\mathrm{HCl}$, the $\mathrm{pH}$ value of the water was first lowered to 1.7. After introducing 1 $\mathrm{mM} \mathrm{FeCl}$, the $\mathrm{pH}$ of the solution was checked again, to find it remained the same as before. It indicates that a dominant form of ion in the solution is $\mathrm{Fe}^{3+}$ because high proton concentration inhibits the formation of ion hydroxide.

Figure 4(a) shows SF spectra from an arachidic acid monolayer in these two conditions. The most striking feature of the data is the enhancement of SF intensity from $\sim 3000 \mathrm{~cm}^{-1}$ to $\sim 3400 \mathrm{~cm}^{-1}$ in $\mathrm{pH} \mathrm{2.6}$. This means that $\mathrm{Fe}(\mathrm{OH})_{3}$ molecules affected the interfacial water structure or $\mathrm{OH}$ stretch mode of $\mathrm{Fe}(\mathrm{OH})_{3}$ itself as seen in the spectra. Previous IR absorption measurement of metal hydroxide in solid argon showed that the $\mathrm{OH}$ stretch mode of $\mathrm{Fe}(\mathrm{OH})_{3}$ is $\sim 3670 \mathrm{~cm}^{-1}$, similar to the value of free (non-hydrogen bonded) $\mathrm{OH}$ of a water molecule [25]. Since $\mathrm{Fe}(\mathrm{OH})_{3}$ will make a hydrogen bonding network with surrounding water molecules, $\mathrm{OH}$ vibrational frequency is expected to be redshifted appreciably. To assign the band in Fig. 4(a), we additionally measured IR absorption spectra of bulk $\mathrm{FeCl}_{3}$ solution. As shown in Fig. 4(b), absorbance of the $0.5 \mathrm{M} \mathrm{FeCl}_{3}$ solution increased from $2800 \mathrm{~cm}^{-1}$ to $3400 \mathrm{~cm}^{-1}$. So, it can be understood that the $\mathrm{OH}$ vibrational mode of $\mathrm{Fe}(\mathrm{OH})_{3}$ contributed to the enhancement of SF intensity in the low frequency $(<3400$ $\mathrm{cm}^{-1}$ ) range. We also tried to observe the IR absorbance change of a $1 \mathrm{mM} \mathrm{FeCl}$ solution, but there was no clear difference, which demonstrates the sensitivity of the sumfrequency spectroscopic technique. Therefore, we concluded that SF intensity enhancement in $\mathrm{pH} 2.6$ came from the $\mathrm{OH}$ stretch mode of $\mathrm{Fe}(\mathrm{OH})_{3}$ and surrounding interfacial water having reconstructed structure. On the other hand, SF spectrum in $\mathrm{pH} 1.7$ shows only a small bump around $3080 \mathrm{~cm}^{-1}$, which is assigned as an $\mathrm{OH}$ vibrational mode of the protonated carboxyl headgroup as assigned by Miranda et al [15]. This SF spectrum is identical to that of

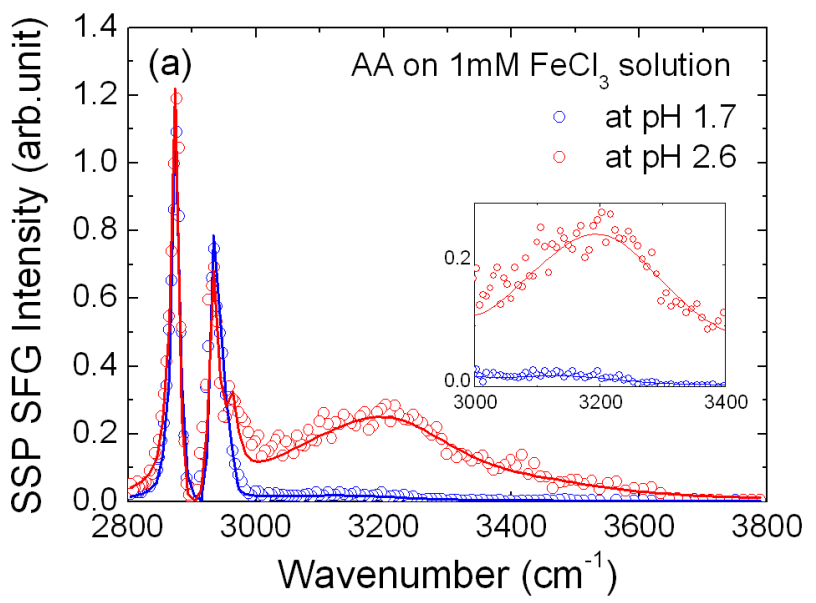

(a)

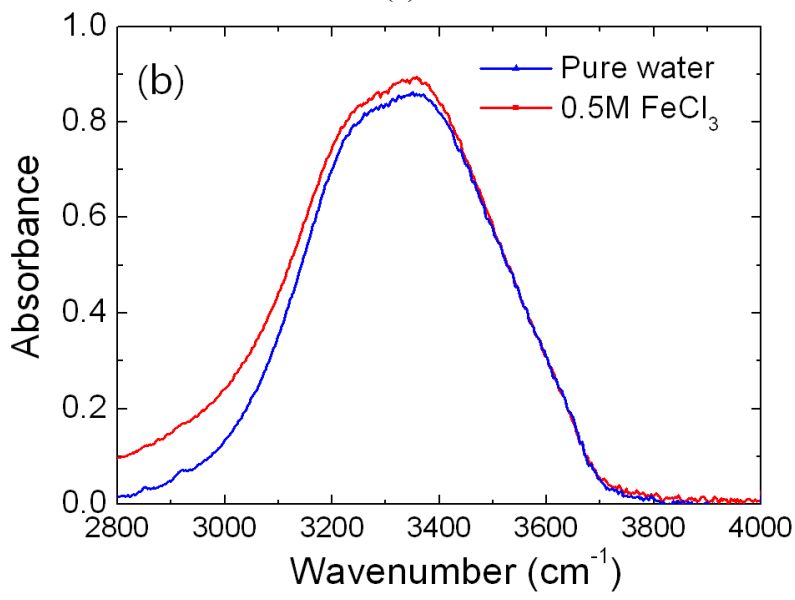

(b)

FIG. 4. (a) SF spectra of arachidic acid monolayer on $1 \mathrm{mM}$ $\mathrm{FeCl}_{3}$ solution. Inset is a blow-up of the original spectrum for showing the existence of a shoulder around $3080 \mathrm{~cm}^{-1}$ at $\mathrm{pH}$ 1.7. Solid lines are fitting curves deduced from Eq. 3. (b) IR absorption spectra of pure water (blue line) and $0.5 \mathrm{M} \mathrm{FeCl}_{3}$ solution (red line).

an arachidic acid monolayer in $\mathrm{pH} 1.5$ pure water, not showing any clear sign of $\mathrm{Fe}^{3+}$ absorption.

As shown in reference spectra in Fig. 2, an arachidic acid monolayer is charge neutral in $\mathrm{pH}$ lower than 3.5. So, it is expected that coulomb interaction cannot promote ion adsorption to the monolayer. Moreover, in the case of $\mathrm{FeCl}_{3}$ solution in $\mathrm{pH} 2.6$, both iron hydroxides and carboxyl head groups have no charges. We supposed that $\mathrm{Fe}(\mathrm{OH})_{3}$ adsorption was related to chemical interaction such as hydrogen or covalent bonding. We would like to suggest that there are strong covalent or hydrogen bonding connection between $\mathrm{Fe}(\mathrm{OH})_{3}$ and the oxygen atom of the carboxyl headgroup, and the remaining $\mathrm{OH}$ moieties of $\mathrm{Fe}(\mathrm{OH})_{3}$ forming a hydrogen bonding network with other $\mathrm{Fe}(\mathrm{OH})_{3}$ ions or water molecules.

For simple trivalent $\mathrm{La}^{3+}$, the adsorption to the interface is due to electrostatic interaction, obviously from negligibly 
small SF signal in the $\mathrm{OH}$ range. SF intensity of the $\mathrm{CH}_{x}$ range was also smaller than that of monolayers on pure water. Even for Fig. 3, monolayer in $\mathrm{pH} 10.2$ shows smaller SF intensity than that in $\mathrm{pH}$ 8.4. So, $\mathrm{La}^{3+}$ ions on the surface seemed to change not only net surface charge density, but monolayer structure. Previous X-ray and imaging ellipsometry studies about an arachidic acid monolayer on $\mathrm{CaCl}_{2}$ solution showed that the portion of spontaneously collapsed bilayer was increased in the presence of multivalent cation in water $[26,27]$. So, the smaller SF intensity in the $\mathrm{CH}_{\mathrm{x}}$ region is considered to come from the bilayer from spontaneous collapse induced by $\mathrm{La}^{3+}$ ions.

\section{CONCLUSION}

To summarize, the adsorption behavior of $\mathrm{La}^{3+}$ and $\mathrm{Fe}$ $(\mathrm{OH})_{3}$ on an arachidic acid monolayer was studied by surface sensitive sum-frequency generation spectroscopy. Adsorption of the $\mathrm{La}^{3+}$ ion is driven by the coulomb interaction. This is the same as the adsorption behavior of other simple mono- and divalent cations as already investigated [28]. $1 \mathrm{mM}$ of $\mathrm{LaCl}_{3}$ seems to be sufficient to make a charge neutral condition of the monolayer over all the $\mathrm{pH}$ range. On the other hand, adsorption of $\mathrm{Fe}(\mathrm{OH})_{3}$ is induced by chemical bonding between iron hydroxide and the carboxyl headgroup.

\section{ACKNOWLEDGMENT}

This work was supported by the National Research Foundation (NRF) grant funded by the Korea government (MEST) No. 2011-0017435 and No. 2011-0031496. DV is supported by the Office of Basic Energy Sciences, U.S. Department of Energy under Contract No. DE-AC0207CH11358.

\section{REFERENCES}

1. D. L. Nelson and M. M. Cox, Principles of Biochemistry, $2^{\text {nd }}$ ed. (W. H. Freeman and Company, New York, NY, USA, 2004).

2. S. V. Perry and T. C. Grey, "A study of the effects of substrate concentration and certain relaxing factors on the magnesium-activated myofibrillar adenosine triphosphatase," Biochem. J. 64, 184-192 (1956).

3. R. Blakemore, "Magnetotactic bacteria," Science 190, 377379 (1975).

4. M. Tanaka, E. Mazuyama, A. Arakaki, and T. Matsunaga, "MMS6 protein regulates crystal morphology during nanosized magnetite biomineralization in vivo," J. Biol. Chem. 286, 6386-6392 (2011).

5. A. Arakaki, J. Webb, and T. Matsunaga, "A novel protein tightly bound to bacterial magnetic particles in magnetospirillum magneticum strain AMB-1,” J. Biol. Chem. 278, 8745-8750 (2003).

6. T. Matsunaga and Y. Okamura, "Genes and proteins involved in bacterial magnetic particle formation," Trends in Microbiology 11, 536-541 (2003).

7. G. Roberts, Langmuir-blodgett Films (Plenum Press, New York and London, 1990).

8. V. M. Kaganer, H. Mohwald, and P. Dutta, "Structure and phase transitions in Langmuir monolayers," Rev. Mod. Phys. 79, 779-819 (1999).

9. S. Hénon and J. Meunier, "Microscope at the Brewster angle: direct observation of first order phase transitions in monolayers," Rev. Sci. Instrum. 62, 936-939 (1991).

10. J. Umemura, T. Kamata, T. Kawai, and T. Takenaka, "Quantitative evaluation of molecular orientation in thin LangmuirBiodgett films by FT-IR transmission and reflection-absorption spectroscopy," J. Phys. Chem. 94, 62-61 (1990).

11. R. W. Boyd, Nonlinear Optics, 2nd ed. (Academic Press, 2003).

12. Q. Du, R. Superfine, E. Freysz, and Y. R. Shen, "Vibrational Spectroscopy of water at the vapor/water interface," Phys. Rev. Lett. 70, 2313-2316 (1993).

13. P. B. Miranda and Y. R. Shen, "Liquid interfaces: a study by sum-frequency vibrational spectroscopy," J. Phys. Chem. B 103, 3292-3307 (1999).

14. J. Sung, K. Park, and D. Kim, "Surface of alcohol-water mixtures studied by sum-frequency generation vibrational spectroscopy," J. Phys. Chem. B 109, 18507-18514 (2005).

15. P. B. Miranda, Q. Du, and Y. R. Shen, "Interaction of water with a fatty acid Langmuir film," Chem. Phys. Lett. 286, 1-8 (1998).

16. M. C. Gurau, E. T. Castellana, F. Albertorio, S. Kataoka, S. M. Lim, R. D. Yang, and P. S. Cremer, "Thermodynamics of phase transitions in langmuir monolayers observed by vibrational sum frequency spectroscopy," J. Am. Chem. Soc. 125, 11166-11167 (2003).

17. G. W. H. Wurpel, M. Sovago, and M. Bonn, "Sensitive probing of DNA binding to a cationic lipid monolayer," J. Am. Chem. Soc. 129, 8420-8421 (2007).

18. M. Smits, M. Sovago, G. W. H. Wurpel, D. Kim, M. Muller, and M. Bonn, "Polarization-resolved broad-bandwidth sumfrequency generation spectroscopy of monolayer relaxation," J. Phys. Chem. C 111, 8878-8883 (2007).

19. J. Sung and D. Kim, "Fast motion of the surface alcohol molecules deduced from sum-frequency vibrational spectroscopy," J. Phys. Chem. C 111, 1783-1787 (2007).

20. S. Ong, X. Zhao, and K. B. Eisenthal, "Polarization of water molecules at a charged interface: second harmonic studies of the silica/water interface," Chem. Phys. Lett. 191, 327335 (1992).

21. W. Sung, S. Seok, D. Kim, C. S. Tian, and Y. R. Shen, "Sum-frequency spectroscopic study of Langmuir monolayers of lipids having oppositely charged headgroups," Langmuir 26, 18266-18272 (2010).

22. X. Zhuang, P. B. Miranda, D. Kim, and Y. R. Shen, "Mapping molecular orientation and conformation at interfaces by surface nonlinear optics," Phys. Rev. B 59, 12632-12640 (1999).

23. Y. R. Shen and V. Ostroverkhov, "Sum-frequency vibrational spectroscopy on water interfaces: polar orientation of water molecules at interfaces," Chem. Rev. 106, 1140-1154 (2006). 
24. W. Wang, R. Y. Park, A. Travesset, and D. Vaknin, "Ionspecific induced charges at aqueous soft interfaces," Phys. Rev. Lett. 106, 056102 (2011).

25. X. Wang and L. Andrews, "Infrared spectra of $\mathrm{M}(\mathrm{OH}) 1,2,3$ $(\mathrm{M}=\mathrm{Mn}, \mathrm{Fe}, \mathrm{Co}, \mathrm{Ni})$ molecules in solid argon and the character of first row transition metal hydroxide bonding," J. Phys. Chem. A 110, 10035-10045 (2006).

26. S. Seok, T. J. Kim, S. Y. Hwang, Y. D. Kim, D. Vaknin, and D. Kim, "Imaging of collapsed fatty acid films at air- water interfaces," Langmuir 25, $9262-9269$ (2009).

27. D. Vaknin, W. Bu, S. K. Satija, and A. Travesset, "Ordering by collapse: formation of bilayer and trilayer crystals by folding Langmuir monolayers," Langmuir 23, 1888-1897 (2007).

28. W. Bu and D. Vaknin, "X-ray fluorescence spectroscopy from ions at charged vapor/water interfaces," J. Appl. Phys. 105, 084911 (2009). 